\title{
Real-time Production Visibility Protocol using Radiofrequency Identification Technology at a Blinds Manufacturing Company
}

\author{
By Rodolfo Ramírez-Hernández ${ }^{*}$ \\ Guillermo Cuamea-Cruz ${ }^{\dagger}$
}

Visibility Systems are utilized in the manufacturing processes to detect the status of the production activity, analyze its performance (such as total production number, cycle time \& bottlenecks) and help make intelligent decisions to improve them. These systems are an essential element of the business strategy and can contribute to obtaining competitive advantage through a real-time visibility characteristic. Radiofrequency Identification (RFID) is an Automated Data Identification and Acquisition Technology that enables the collection and transferring of production and business information for the purpose of managing and presenting manufacturing data in a way that can generate added value to the industry of application. This technology uses radio waves in order to transfer the previously recorded data from an electronic tag (RFID tag) that is adhered to an object or product, through a transceiver (RFID reader) for the purpose of identifying and tracing the object of interest through the manufacturing process utilizing a backend system for the filtering and administrating of such coded information. This paper introduces to the basics of the RFID technology and applies this knowledge to a Manufacturing Process in a company located in Mexico through the development of an RFID Application Protocol. The RFID Application Protocol consists of the following parts: RFID Architecture Selection guided by key questions; RFID Traceability Network Design including the Dynamic of the Product; RFID Application Parameters through experimentation in an RFID Laboratory; and finally Manufacturing Data Visibility through Simulation.

\section{Introduction}

Many manufacturing companies have adopted new information systems in order to monitor manufacturing activities and take immediate action to solve any emerging event that could cause a disruption in production.

*Full Time Master Student, Universidad de Sonora, México.

${ }^{\dagger}$ Full Professor, Universidad de Sonora, México. 
Radiofrequency Identification (RFID) is a technology that has proved to be a great candidate for tracking and tracing of manufacturing goods in the last decades.

RFID architecture consists of RFID tags, RFID readers and a backend system that manages the produced data for its communication. Deciding the architecture that best suits the needs of the company contributes to the success of an implementation. RFID Traceability Network refers to the arrangement of the RFID components that will enable the tracking \& tracking of goods. A correct RFID Network design considering the location of RFID readers will also prevent the application from failing.

The purpose of this article is to develop a Real-time Production Visibility Protocol in a Manufacturing Company from the Problem Statement to providing Application Parameters resulting from Experimentation in an RFID Laboratory.

\section{Visibility Systems (VS)}

VS are utilized in the manufacturing process for detecting its state, analyze its performance and help make intelligent decisions to improve it (Monette \& Van Bogart, 2009). At the same time, the VS provide a complete history of the product cycle it order to contribute to more precise solutions towards a more efficient production flow and delivery of final products. Such systems are an essential element for accomplishing strategic objectives and the desired competitive advantage through faster manufacturing time, on-time deliver and production efficiency increasing through a real-time feature (Rönkkö, 2006). In typical VS, it is possible to accurately determine when and where a product was built by scanning a serial number.

\section{Radio Frequency Identification (RFID) Technology}

Radio Frequency Identification is an automatic data identification and acquisition system that allows collecting and transferring production and business information (Ostojic et al., 2008). In a more technical way, RFID is a technology that uses radio waves in order to transfer information from an electronic tag, called RFID tag, adhered to an object, through a reader/antenna for the purpose of identifying and tracking objects (Chiagozie \& Nwaji, 2012).

In recent years, RFID has drawn the attention of the manufacturing industry and other businesses (Ngai et al., 2008). On par with significant technological improvements, greater availability of components, cost reduction and shared best practices, the technology has proven its value in the significant improvement in operational efficiency which has driven its widespread acceptance in the industrial field (Chiagozie \& Nwaji, 2012). 


\section{RFID System Architecture}

A typical RFID System Architecture consists of three main components: RFID tag, RFID readers and a Backend System (Chen et al., 2007).

RFID tags: are labels adhered to the objects of interest. The tag contains a chip that storages identification data through an electronic code. When the label passes through an electromagnetic field generated by the RFID reader, the tag communicates the identification data back to the reader (Wu et al., 2011).

RFID readers: the function of the readers is that of generating an electromagnetic field in order to excite the tags (when passive ones are used) and facilitate the communication among them. RFID readers (or their antennas) are placed in strategic locations so that they can detect the tags more accurately (Wu et al., 2011).

Backend System: RFID readers are connected to a computational network where the data is collected and processed (Chen et al, 2007). This system is responsible for coding and decoding the electronic information written in the RFID tags, filter this information, store it and then share it to the proper communication channels of the industry.

\section{RFID Traceability Network (RFIDTN)}

With an RFIDTN assets can be monitored in a precise and automatic manner. Moreover, their historical paths can be recorded in real-time. Traceability improves the production rate and quality of the product deriving on better business decisions. The components of an RFIDTN according to Ranasinghe et al. (2011) are the following:

Node: represents a point of observation. Each node is related to the installation of an RFID reader.

Object: represents a uniquely identified tagged article with an RFID tag.

Connection: is a link between nodes. It refers to the way the different RFID readers communicate with each other.

Network: is a set of composite connections that represents the relation between nodes. The related information gathered in the network will be then shared with a backend system that will manage it.

Contention: a main object containing one or more secondary objects (such as subassemblies or packaging).

Finally, the dynamics that follow the articles traveling in an RFIDTN are related to their movements, the amount of time they remain at a node and the path they follow throughout the manufacturing process. 


\section{Problem Statement}

The company where the RFID protocol takes place is a Blinds Manufacturer located in Mexico. Its objective market is positioned in the United States and Canada having clients such as Wal-Mart ${ }^{\circledR}$ and Lowe's ${ }^{\circledR}$.

In the field of visibility of the production entities insufficient advances have been made. The only spots for visualizing the location and flow of assets are at the beginning and at the end of the process. There are frequent bottlenecks, product specification changes and a big gap on the real status of the production system at a certain time.

RFID seems to be a good choice for solving the previous issues related to manufacturing information in a timely manner for decision making purposes in order to improve the manufacturing process.

The next part will deal with the followed methodology of application considering such technology.

\section{Methodology}

General introductions to RFID technology in the context of Visibility of Production assets and the problematic in a real industry have taken place. Given this scenario, an integration of the conceptual diversity previously presented will be generated through a proposed Methodology.

\section{What RFID Architecture to use}

The first part consists on deciding which RFID Architecture best suits the characteristics of the product and its manufacturing system. For this purpose, a series of key questions will be answered.

What kind of RFID frequency is needed? Since the blinds are distributed to the U.S.A. and Canada the UHF (Ultra High Frequency) at $902-928 \mathrm{MHz}$ is the one to be utilized (Kommandur, 2004).

What are the characteristics of raw material? Blinds are manufactured with $95 \%$ of dry wood (such as bamboo and reed), fabric and nylon (Levolor, 2012). According to Sanghera, (2007) \& Lee et al., (2009) the frequency that meets these needs continues to be UHF.

What are the dimensions of the products? According to Levolor (2012), the smallest product that can be manufactured is $25.4 \mathrm{~cm}$ wide. So the RFID tags must be smaller than the narrowest blind.

What are the dimensions of the production floor? The total area is $667 \mathrm{~m}^{2}$ $(23 \times 29 \mathrm{~m})$. This information will be used to obtain the number of RFID readers needed to cover the entire area.

What type of RFID reader to install? It is important that the RFID reader and RFID tags work in the same frequency. So UHF RFID readers must be used. On the other hand, for the given purposes, fixed readers are recommended (Blanch, 2007). 


\section{RFIDTN Design}

An RFIDTN Design is proposed based on Ranasinghe et al. (2011) \& Chen et al. (2011).

In application terms, nodes translate into the location and installation of an RFID reader/antenna. UHF RFID readers have a reading range of about $9 \mathrm{~m}$ (Blanch, 2007). Considering this information, the production layout is then divided into $1 \mathrm{~m}^{2}$ blocks and the coordinates for each node are obtained considering a uniform location.

Objects are the blinds to be detected. UHF RFID tags are used to label each object.

Connections \& Network. A closed-loop network will be used for the RFIDTN Design since no data sharing is necessary with other parts of the supply chain. Each RFID Reader will be connected with one another throughout the production layout.

Path. This data is obtained from Flowchart Diagrams available in SAP ${ }^{8}$ reports. This information represents the operations/stations each product has to visit in order to be manufactured.

Time. Time Study procedures have been applied by the company and are also available in $\mathrm{SAP} \AA$ reports.

Contention. In real terms, contention is related to the packing of the blinds, which is one of the last operations of the manufacturing process.

Figure 1 shows the complete RFIDTN Design considering the previous data.

Figure 1. RFID Traceability Network Design \& RFID Reader Coordinates

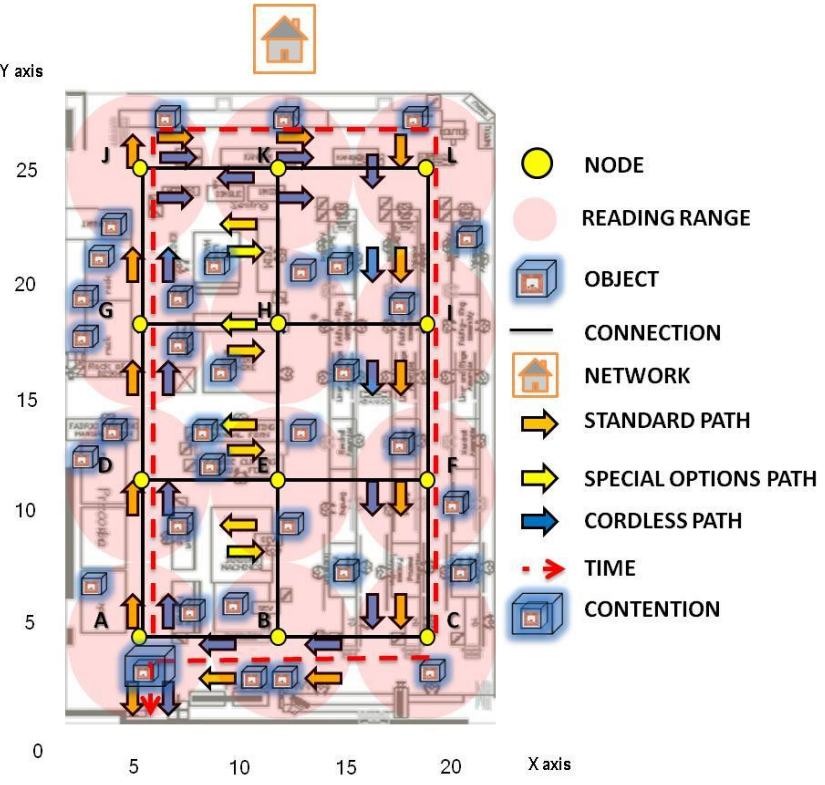

\begin{tabular}{|c|c|}
\hline RFID READER & XY COORDINATES \\
\hline A & $(4,3.7)$ \\
\hline B & $(11.5,3.7)$ \\
\hline C & $(19.2,3.7)$ \\
\hline D & $(4,10.7)$ \\
\hline E & $(11.5,10.7)$ \\
\hline F & $(19.2,10.7)$ \\
\hline G & $(4,18.2)$ \\
\hline H & $(11.5,18.2)$ \\
\hline I & $(19.2,18.2)$ \\
\hline J & $(4,25.7)$ \\
\hline K & $(11.5,25.7)$ \\
\hline L & $(19.2,25.7)$ \\
\hline
\end{tabular}

Optimal Application Parameters

The location of RFID readers has already been solved on the XY coordinates. There is still a need for finding the optimal height and RFID reader power that ensure the detection of the blinds throughout the 
manufacturing process. For this purpose an Experiment was designed at the RFID Lab at the University of Parma, IT.

The materials used in this experiment were the following: 1 sample blind $(25.3$ X $30.48 \mathrm{~cm}), 3$ smartrac dogbone and 3 smartrac web Passive RFID UHF EPC Class 1 Gen2 tags (860-960 MHz), 1 Laird Technologies RFID Antenna (865-960 MHz, circular polarization, 10 watts), 1 Impinj Speedway RFID Reader (4 antenna inputs), RSA ${ }^{\circledR}$ Interface, Microsoft Excel ${ }^{\circledR} \&$ a Lap Top.

The experiment consists of an arrangement of 1 RFID Antenna and 1 RFID Reader installed in a tower and a blind that is read at different distances. The RFID Reader is connected to the RFID antenna and to the Lap Top that will make possible the communication of the components through RSA ${ }^{\circledR}$ interface via wireless. Blinds are then individually tagged at 3 different locations with the 2 types of RFID tags. 30 repetitions were performed for each type of tag, the selected RFID powers and Distances (heights) between the product and the RFID Reader. Each reading lasts 3s and then a Microsoft Excel (B) datasheet generates automatically. All of the above is in accordance to RFID Lab (2012). Figure 2 shows the Experiment Layout along with some pictures of the actual process. Figure 3 summarizes the results obtained from the measuring activity.

Figure 2. Experiment Layout and Illustrations

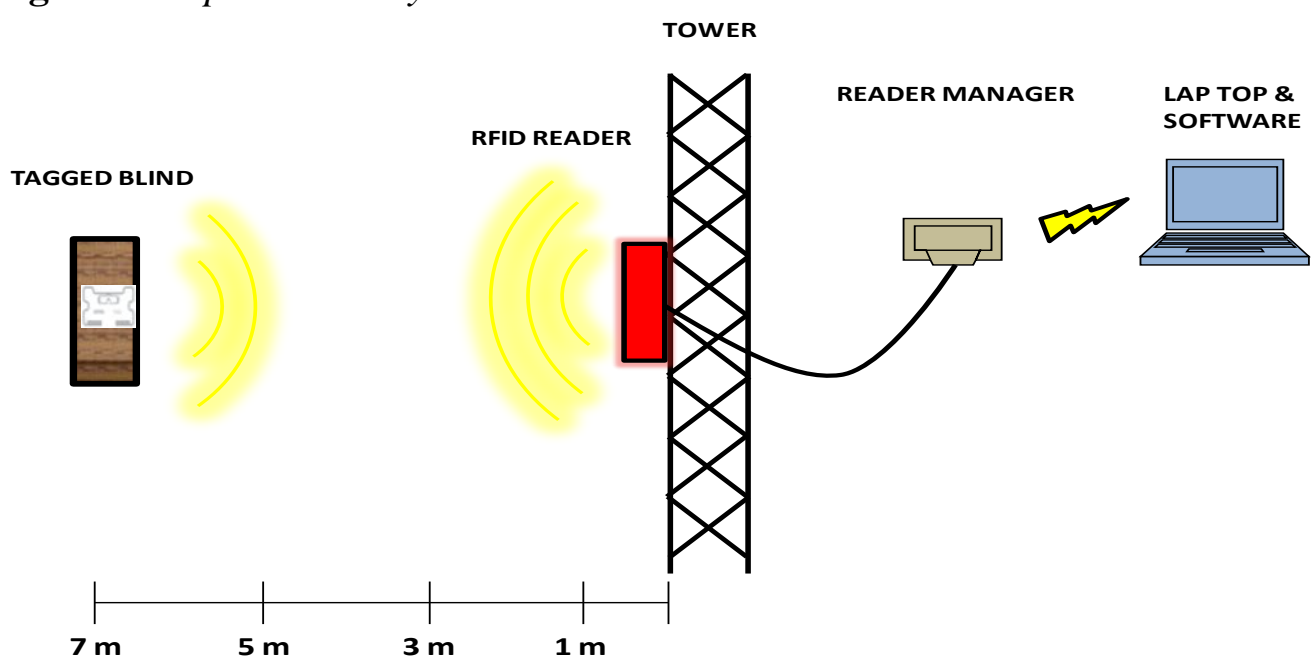

HEIGHTS

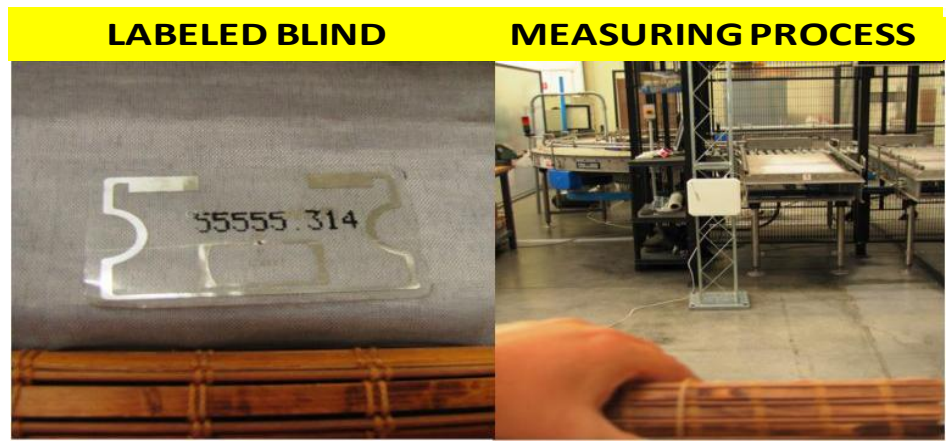


Figure 3. Tags Detection at Different RFID Reader Powers


\section{Manufacturing Data Visibility through Simulation}

Finally, the present experiment aims to detect the product, simulate its manufacturing process and obtain visibility data through the installed RFID infrastructure.

The list of materials used is the following: 1 sample blind (45.72 X 60.96 $\mathrm{cm}$ ), 3 Smartrac Dogbone Passive RFID UHF EPC Class 1 Gen2 tags (860-960 MHz), 2 Laird Technologies RFID Antennas (865-960 MHz, circular polarization, 10 watts), 1 Impinj Speedway RFID Reader (4 antenna inputs), 1 
processes conveyor, 1 chronometer, RSA ${ }^{\circledR}$ Interface, Microsoft Excel ${ }^{\circledR}$ \& a Lap Top.

Figure 4. Simulation Experiment Layout TOWER



The experiment layout is presented in figure 4. The sample blind is identified at 3 different locations using the Smartrac Dogbone tags. 2 RFID antennas are installed representing the different locations of the manufacturing area. The power used is $27.2 \mathrm{dBm}(0.525 \mathrm{~W})$ at $3 \mathrm{~m}$ from the product in accordance to the previous experiment results. The blind is then transported from one location to the other and stopped each time emulating the standard time for each operation. The obtained data simulates the information gathering of a real manufactured blind. This data is then managed through RSA ${ }^{\circledR}$ interface and exported to Microsoft Excel ${ }^{\circledR}$, compared to the SAP Reports so that performance metrics can be established (see figure 5). The visibility and location of assets is then achieved in real time in a simulation environment.

Figure 5. Real-time Visibility Data Comparison

\begin{tabular}{|c|c|c|c|c|c|c|c|c|c|}
\hline \multicolumn{2}{|c|}{ SAP TIME } & \multicolumn{2}{|c|}{ REAL TIME } & \multirow{2}{*}{$\begin{array}{l}\text { DIFFERENCE } \\
\text { PERCENTAGE }\end{array}$} & \multirow{2}{*}{\multicolumn{5}{|c|}{ SAP VS REAL TIME }} \\
\hline SEC & MIN & SEC & MIN & & & & & & \\
\hline 14.4 & 0.24 & 15.43 & 0.257 & $7.17 \%$ & $\begin{array}{l}\text { PACKAGING } \\
\text { FINAL INSP. }\end{array}$ & & & & \\
\hline 5.4 & 0.09 & 5.85 & 0.098 & $8.39 \%$ & CORDING & & & & \\
\hline 33.12 & 0.552 & 36.79 & 0.613 & $11.08 \%$ & RING ASSY & - & 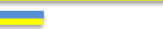 & & \\
\hline 27.36 & 0.456 & 26.65 & 0.444 & $2.57 \%$ & FINISHING & & & & \\
\hline 203.4 & 3.39 & 213.58 & 3.560 & $5.00 \%$ & BLIND ASSY & $=$ & & & \\
\hline 241.92 & 4.032 & 237.85 & 3.964 & $1.68 \%$ & BOTTOM ASSY & & & & \\
\hline 39.96 & 0.666 & 40.12 & 0.669 & $0.41 \%$ & $\begin{array}{l}\text { VALLASSY } \\
\text { SAERYCDD }\end{array}$ & & & & \\
\hline 41.76 & 0.696 & 43.24 & 0.721 & $3.54 \%$ & HEADRAIL CUT & 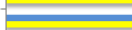 & & $=$ & \\
\hline 33.12 & 0.552 & 33.78 & 0.563 & $1.98 \%$ & BINDING & 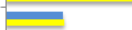 & & & \\
\hline 150.84 & 2.514 & 142.10 & 2.368 & $5.79 \%$ & FABRIC CUT & 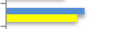 & & & \\
\hline 105.12 & 1.752 & 105.67 & 1.761 & $0.53 \%$ & BUND CUT & 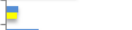 & & & \\
\hline 169.92 & 2.832 & 233.44 & 3.469 & $37.38 \%$ & PRECUT & $\rightleftharpoons$ & & & \\
\hline 111.6 & 1.86 & 193.77 & 3.229 & $73.63 \%$ & & 50 & 150 & 200 & 250 \\
\hline 116.64 & 1.944 & 112.89 & 1.881 & $3.22 \%$ & mREAL TIME & & TIME (SECONDS) & & \\
\hline 1294.56 & 21.576 & 1441.16 & 23.598 & $9.37 \%$ & SAP TIME & & & & \\
\hline
\end{tabular}




\section{Conclusions \& Future Works}

This study has established UHF as the best RFID Architecture for implementing a Real-time Production Visibility System in the Blinds Sector.

RFIDTN Design has also been presented. A uniform layout of the RFID Readers with their corresponding location has been obtained.

Optimal Application Parameters have been calculated through experimentation at an RFID Lab using the installed technology and sample product. The maximum height an RFID Reader/Antenna can be placed ensuring the detection of the product is $7 \mathrm{~m}$ at $100 \%$ of power using Smartrac Dogbone RFID Tags.

RFID Technology enabled the data gathering and visibility of the status of the product during manufacturing as the simulation shows. This information can be used as Decision Making auxiliary for improvement purposes.

Optimal placement of RFID Readers considering key factors such as Optimal Tag Coverage and Interference between RFID Readers is necessary to obtain a closer-to-reality approach. Particle Swarm Optimization Algorithms have demonstrated to have good results for solving such Optimization Problems.

\section{References}

Blanch, B. (2007). 'RFID Technology Application in the Supply Chain'. M. Sc. diss., Barcelona University. [In Catalan]

Chen, J.L., Chen, M.C., Chen, C.W. y Chang, Y.C. (2007). 'Architecture design and performance evaluation of RFID object tracking systems'. Computer Communications 30: 2070-2086.

Chiagozie, O. y Nwaji, O. (2012). 'Radio Frequency Identification (RFID) based Attendance System with Automatic Door Unit'. Academic Research International 2(2): 168-183.

Kommandur, B. (2004). 'Exploration of Disruptive Technologies for low cost RFID Manufacturing'. M. Sc. diss., Massachusetts Institute of Technology.

Lee, W., Cheung, B. \& Kwok, S. (2009). 'Industrial Automation: Digital Manufacturing and RFID-Based Automation'. In: S.Y. Nos (ed.), Springer Handbook of Automation. Part F: Industrial Automation, 859-879. Berlin: Springer Berlin Heidelberg.

Levolor Kirsch Window Fashions (2012). Custom Natural Shades, Suggested Retail Pricing. Levolor ${ }^{\circledR}$.

Monette, F. \& Van Bogart, M. (2009). The business case for Track, Trace, and Control. Microsan Systems, Inc.

Ngai, E.W.T., Moon, K.K.L., Riggins, F.J. \& Yi, Y.C., (2008). 'RFID research: an academic literature review (1995-2005) and future research directions'. International Journal of Production Economics 112 (2): 510-520.

Ostojic, G., Lazarevic, M., Stankovski, S., Cosic, I. \& Radosavljevic, Z. 
(2008). 'Radio Frequency Identification Technology Application in Disassembly Systems'. Journal of Mechanical Engineering 54 (11): 759767.

Ranasinghe, D.C., Harrison, M., Främling, K., McFarlane, D. (2011). 'Enabling through life product instance management: solutions and challenges'. Journal of Network Computing Applications 34 (3): 1015-1031.

RFID Lab (2012). RFID 4 Fashion Certified Program: Report Test. Parma: University of Parma.

Rönkkö, M. (2006). 'A model for item centric material control in manufacturing'. M. Sc. diss., Helsinki University of Technology.

Sanghera, P. (2007). RFID+ Study Guide and Practice Exam. Rockland: Syngress Publishing, Inc.

Wu, J., Ranasinghe, D., Sheng, Q., Zeadally, S. \& Yu, J. (2011). 'RFID enabled traceability networks: a survey'. Distributed Parallel Databases 29: 397443. 